BMJ Open

Diabetes

Research

\& Care

\section{Placental proteome abnormalities in women with gestational diabetes and large-for-gestational-age newborns}

To cite: Assi E, D'Addio F, Mandò C, et al. Placental proteome abnormalities in women with gestational diabetes and large-forgestational-age newborns. BMJ Open Diab Res Care 2020;8:e001586. doi:10.1136/ bmjdrc-2020-001586

- Supplemental material is published online only. To view please visit the journal online (http://dx.doi.org/10.1136/ bmjdrc-2020-001586).

EA and FD are joint first authors.

Received 18 May 2020 Revised 24 September 2020 Accepted 12 0ctober 2020

Check for updates

(c) Author(s) (or their employer(s)) 2020. Re-use permitted under CC BY-NC. No commercial re-use. See rights and permissions. Published by BMJ.

For numbered affiliations see end of article.

Correspondence to Dr Paolo Fiorina; paolo.fiorina@childrens. harvard.edu

\begin{abstract}
Introduction Gestational diabetes mellitus (GDM) is the most frequent metabolic complication during pregnancy and is associated with development of short-term and long-term complications for newborns, with largefor-gestational-age (LGA) being particularly common. Interestingly, the mechanism behind altered fetal growth in GDM is only partially understood.
\end{abstract}

Research design and methods A proteomic approach was used to analyze placental samples obtained from healthy pregnant women $(n=5)$, patients with GDM $(n=12)$ and with GDM and LGA $(n=5)$. Effects of altered proteins on fetal development were tested in vitro in human embryonic stem cells (hESCs).

Results Here, we demonstrate that the placental proteome is altered in pregnant women affected by GDM with LGA, with at least 37 proteins differentially expressed to a higher degree $(p<0.05)$ as compared with those with GDM but without LGA. Among these proteins, 10 are involved in regulating tissue differentiation and/ or fetal growth and development, with bone marrow proteoglycan (PRG2) and dipeptidyl peptidase-4 (DPP-4) being highly expressed. Both PRG2 and DPP-4 altered the transcriptome profile of stem cells differentiation markers when tested in vitro in hESCs, suggesting a potential role in the onset of fetal abnormalities.

Conclusions Our findings suggest that placental dysfunction may be directly responsible for abnormal fetal growth/development during GDM. Once established on a larger population, inhibitors of the pathways involving those altered factors may be tested in conditions such as GDM and LGA, in which therapeutic approaches are still lacking.

\section{INTRODUCTION}

Gestational diabetes mellitus (GDM), which affects $3 \%-25 \%$ of pregnancies, is defined as glucose intolerance diagnosed in pregnancy ${ }^{1}$ and is associated with the risk of developing adverse pregnancy outcomes. ${ }^{2}$ Fetal growth abnormalities are frequent in GDM, with large-for-gestational-age (LGA) being the most common, while restricted fetal growth is also observed but to a lesser extent. ${ }^{3}$ Because

\section{Significance of this study}

What is already known about this subject?

- Fetal growth abnormalities are frequent in gestational diabetes mellitus (GDM), with large-forgestational-age (LGA) being the most common.

- Placenta in GDM showed alterations in pathways linked to inflammation, oxidative stress, hypoxia, insulin resistance and metabolic complications.

What are the new findings?

- Placental proteome is dysregulated in GDM, particularly in those with LGA.

- Upregulation of proteoglycan (PRG2) and dipeptidyl peptidase-4 (DPP-4) was evident in GDM/LGA cohort.

- PRG2 and DPP-4 alter the expression of genes relevant for human embryonic stem cells (hESCs) differentiation towards cardiac, osteogenic and endodermic progenitors.

How might these results change the focus of research or clinical practice?

- PRG2 and/or DPP-4 upregulation may be involved in fetal abnormalities in GDM.

the placenta is the major organ responsible for fetal nourishment and growth, any alterations in the placental phenotype or function may have an impact on the fetus. ${ }^{45}$ Identifying new factors altered within the placenta and determining whether they affect fetal growth and health will help to clarify the pathological role of placental changes in GDM. This may also pave the way for new successful strategies in preventing, managing and treating maternal and fetal complications in GDM pregnancies. A proteomic approach has been previously employed in disease conditions, including those related to pregnancy, to screen for major molecules and pathways involved in tissue/organ damage ${ }^{6-9}$ However, 
data available on the placental proteomic profile in GDM are scarce and primarily related to maternal glucose intolerance effects on placental pathways associated with insulin resistance, metabolic alterations and inflammatory/oxidative stress. ${ }^{81011}$ Here, we explore whether the placental proteome is altered in GDM, particularly in those with LGA, with a specific focus on factors that may directly or indirectly control fetal growth.

\section{RESEARCH DESIGN AND METHODS}

Sample collection and protein extraction

Placental tissues were collected from GDM with appropriate for gestational age (GDM), GDM with LGA (GDM/LGA) and healthy pregnant women (controls) who gave birth by elective caesarean section and provided informed consent according to standard operating procedures. GDM was defined based on the International Federation of Gynecology and Obstetrics guidelines. ${ }^{12}$ LGA was defined as birth weight greater than the 90th percentile according to gestational age and sex. Normal pregnancies (controls) were defined as healthy mothers with normal body mass index (BMI), pregnancy without obstetric complications, and normal fetal growth with birth weight between the 10th and the 90th percentile for Italian references. ${ }^{12}$ Proteins were extracted from $100 \mathrm{mg}$ of placental tissue washed in Phosphate Buffered Saline (PBS) and minced into small pieces. Selected chorionic villi were then disrupted in liquid nitrogen and solubilized in $500 \mu \mathrm{L}$ RIPA lysis buffer supplemented with protease inhibitor cocktail.

\section{Liquid chromatography-tandem mass spectrometry (LC-MS/ MS) analysis}

Ten-microliter lysate from each sample were pooled for GDM, GDM/LGA and controls, and total protein concentration was measured. Forty micrograms of total protein from each sample was in-solution digested as previously described. ${ }^{13}$ Samples were desalted, and mass spectrometry analysis was performed in technical triplicates using Q-Exactive mass spectrometer equipped with a nanoelectrospray ion source. All MS/MS samples were analyzed using the Mascot search engine to search the UniProt Human Proteome20180523 (93 164 sequences, 37039 836 residues). Raw data were loaded into the MaxQuant software V.1.6.1.0, and label-free protein quantification was based on the intensities of precursors. Peptides and proteins were accepted with an false discovery rate $>1 \%$, with two minimum peptides per protein.

\section{Bioinformatics analysis}

The complete dataset of proteins was analyzed by Student's t-test using $\mathrm{MeV}$ software V.4.9.0. Proteins significantly different $(p<0.001)$ in the controls versus GDM pool and in GDM versus GDM/LGA $(\mathrm{p}<0.05)$ were further subjected to hierarchical clustering analysis. Search Tool for the Retrieval of Interacting Genes (STRING) V.10.5 database was used to search for protein-protein networks of differentially expressed proteins (DEPs) and later visualized in Cytoscape V.3.6.1; the BINGO plugin of Cytoscape was used for gene ontology enrichment. ${ }^{14}$

\section{Cell culture}

hESCs were a generous gift of Professor Thorsten Schlaeger lab from Harvard Medical School and were cultured in feeder independent medium (mTeSR1) on BD Matrigel hESC-qualified Matrix coated plate and maintained undifferentiated. Cells were cultured with/ without human recombinant bone marrow proteoglycan (PRG2) $(25 \mu \mathrm{g} / \mathrm{mL}$ ) and dipeptidyl peptidase-4 (DPP-4) (100 ng/mL) for 72 hours and then collected for RNA extraction. Beta-lox5 human beta cell line was a generous gift from Professor Clayton Mathews, Department of Pathology, Immunology and Laboratory Medicine, University of Florida College of Medicine and were cultured with/without human recombinant DPP-4 at the concentration of $100 \mathrm{ng} / \mathrm{mL}, 500 \mathrm{ng} / \mathrm{mL}$ and $1 \mu \mathrm{g} / \mathrm{mL}$ for 72 hours.

\section{Transcriptome profiling}

Total RNA was isolated from hESC using the RNeasy Mini Kit (Qiagen, Valencia, California, USA) with on-column DNase I digestion. Next, $2 \mu \mathrm{g}$ total RNA from each sample was reverse-transcribed using the RT2 First Strand kit (Qiagen) as previously described. ${ }^{15}$ We used the Human Stem Cell Transcription Factors RT2 Profiler PCR Arrays (PAHS-501Z) (Qiagen). The Profiler PCR Arrays measure quantitatively the expression of a panel of genes using SYBR Green-based real-time PCR. The list of genes is shown in online supplemental table 1. Statistical analysis was performed by using the software available RT2 profiler PCR Array Data Analysis (Qiagen).

\section{Quantitative reverse transcriptase PCR (qRT-PCR)}

RNA from hESC and beta-lox5 was extracted using Trizol Reagent (Invitrogen), and qRT-PCR analysis was performed using TaqMan assays (Life Technologies, Grand Island, New York, USA) according to the manufacturer's instructions. qRT-PCR data were normalized for the expression of ACTB, and $\Delta \Delta \mathrm{Ct}$ values were calculated. Statistical analysis compared gene expression across all cell populations via one-way analysis of variance (ANOVA) followed by Bonferroni post-test for multiple comparisons between the population of interest and all other populations. The complete list of primers used is in online supplemental table 2).

\section{ELISA assay}

DPP-4 and PRG2 levels in placental lysates of all groups of subjects were assessed using commercially available ELISA kits, according to manufacturer's instructions (Mybiosource MBS760997-96 and MBS700931-96).

\section{Statistical analysis}

We determined that a sample size set at four would provide the proteomic study with $80 \%$ power to detect a difference of $30 \%$ in the protein expression between the two groups, with a significance level of $\alpha=0.05$. Other data 
Table 1 Baseline demographic characteristics of healthy pregnant women (CTRL), pregnant women with normal gestational age (GDM) and with LGA (GDM/LGA) enrolled in the study (mean \pm SEM)

\begin{tabular}{|c|c|c|c|c|}
\hline & $\begin{array}{l}\text { CTRL group } \\
(n=5)\end{array}$ & $\begin{array}{l}\text { GDM group } \\
(n=12)\end{array}$ & $\begin{array}{l}\text { GDM/LGA group } \\
(n=5)\end{array}$ & $P$ value \\
\hline \multicolumn{5}{|l|}{ Maternal parameters } \\
\hline Maternal pregravid BMI $\left(\mathrm{kg} / \mathrm{m}^{2}\right)$ & $21.8 \pm 0.6$ & $33.9 \pm 0.8$ & $38.3 \pm 1.6$ & $\begin{array}{l}p<0.0001^{\star} \# \\
p<0.05 \dagger\end{array}$ \\
\hline Gestational weight gain $(\mathrm{kg})$ & $12 \pm 2.2$ & $7.6 \pm 1.5$ & $2.8 \pm 3.8$ & n.s. \\
\hline Maternal age (years) & $36.4 \pm 1.7$ & $35.1 \pm 1.4$ & $34.8 \pm 1.7$ & n.s. \\
\hline Gestational age (weeks) & $39.2 \pm 0.1$ & $39.1 \pm 0.04$ & $39.3 \pm 0.1$ & n.s. \\
\hline Mode of delivery & CS 5 & CS 12 & CS 5 & n.s. \\
\hline Placental weight (g) & $491.4 \pm 30.8$ & $508 \pm 26.8$ & $530.0 \pm 50.7$ & n.s. \\
\hline Fasting glucose at delivery (mg/dL) & $78.2 \pm 4.6$ & $84.1 \pm 3.2$ & $100.8 \pm 4.3$ & $p<0.05^{\# \dagger}$ \\
\hline \multicolumn{5}{|l|}{ Fetal parameters } \\
\hline Birth weight (g) & $3606.0 \pm 142.2$ & $3313.0 \pm 76.03$ & $3958.0 \pm 60.0$ & $\mathrm{p}<0.001^{*}$ \\
\hline F/P ratio & $7.4 \pm 0.3$ & $6.7 \pm 0.4$ & $7.8 \pm 0.8$ & n.s. \\
\hline Gender (F/M) & $3 / 2$ & $6 / 6$ & $4 / 1$ & n.s. \\
\hline
\end{tabular}

* and \# This represents statistical significance of CTRL versus GDM and GDM/LGA, respectively.

†This represents statistical significance of GDM versus GDM/LGA.

BMI, body mass index; CS, elective caesarean section; F/P ratio, fetal weight/placental weight ratio; GDM, gestational diabetes mellitus;

LGA, large-for-gestational-age; n.s., not significant.

are presented as mean and SEM. Significance between the two groups was determined by two-tailed unpaired Student's t-test. For multiple comparisons, the ANOVA test with Bonferroni correction was employed. Graphs were generated using GraphPad Prism V.8.2 (GraphPad Software, La Jolla, California, USA). All statistical tests were performed at the $5 \%$ significance level.

\section{RESULTS}

\section{Patient characteristics}

The characteristics of the study populations are summarized in table 1 . No differences were found with regard to maternal or gestational ages, or in placental or fetal weights in GDM versus healthy controls. Pregnant women with GDM showed higher pregravidic BMI but lower weight gain during pregnancy as compared with controls (table 1). The GDM/LGA group displayed higher fetal weight as compared with GDM, while no differences were found in maternal/gestational age, in placental weight or fetal/placenta weight ratio (table 1 ).

\section{The GDM proteome profile}

In order to discover novel factors that may play a role in placental function and fetal development, we first compared the placental proteomes of control and GDM subjects using an unbiased proteomics approach. Mass spectrometry identified 2103 proteins within the placenta, and MaxQuant analysis revealed a unique expression profile in GDM, consisting of 64 DEPs in GDM as compared with controls $(\mathrm{p}<0.001), 24$ of which were downregulated and 40 of which were upregulated (figure 1A). Analysis of the network involving the 64
DEPs using STRING/Cytoscape showed 62 nodes and 90 edges (figure 1B). To discover possible signalling pathways affected by GDM, we underwent these 64 proteins to gene ontology enrichment analysis of biological process (GOBP) with the BINGO plug-in in Cytoscape. While some of these primarily exert protective effects on trophoblasts both in structure and function (eg, invasion, angiogenesis, oxidative stress, barrier and energy support), others were involved in fetal development (iron and nutrient transport, muscle differentiation and nutrients) and protection from fetal loss (anticoagulant and regulation of epithelial-to-mesenchymal transition). However, the aforementioned analysis did not reveal a clear molecular signature strictly related to abnormal fetal growth.

\section{Alterations in fetal growth-related pathways in placentas of GDM with LGA}

Identification of placental factors associated with LGA may provide new insights to design novel diagnostic/therapeutic strategies aimed at managing such severe fetal complications. To this end, we next compared placental proteomes of GDM/LGA and GDM using the aforementioned proteomics approach. Among the 1514 identified proteins, 37 were differentially expressed in GDM/LGA as compared with GDM $(\mathrm{p}<0.05)$, with 22 proteins downregulated and 15 upregulated (figure 2A). GOBP analysis revealed that 10 of these proteins are primarily involved in placental inflammation (eg, placental inflammosome, complement regulation, control of nitric oxide and histamine levels during pregnancy), fetal nutrient supply (lipid metabolism) and hormonal homeostasis (cortisol 


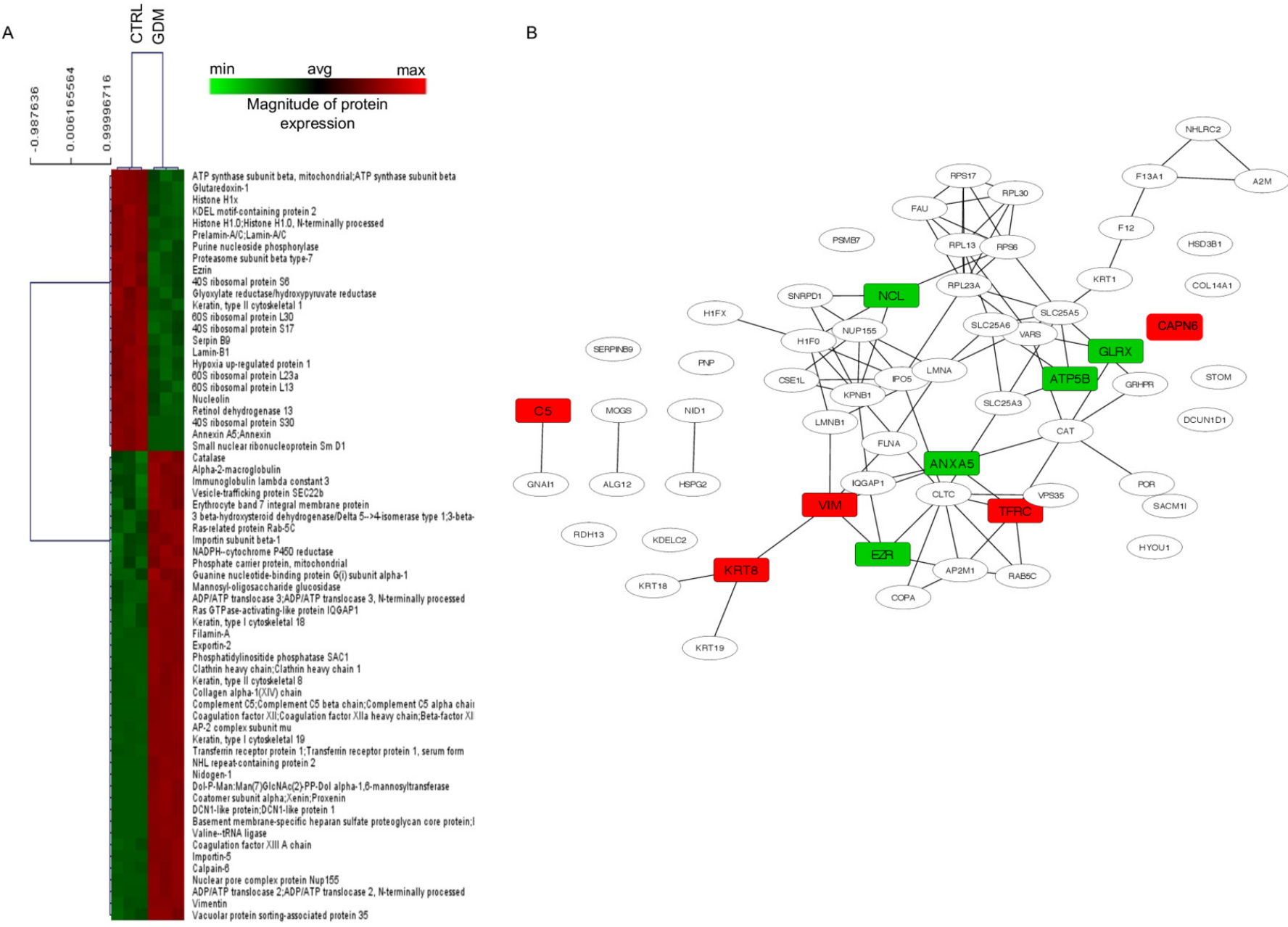

Figure 1 Placental proteomic profile of GDM versus CTRL women. (A) Heat MAP representing the placental proteomic profile of women with GDM as compared with that of healthy subjects (CTRL). The complete dataset of identified and quantified proteins was subjected to statistical analysis $(p<0.001)$. Significantly differentially expressed proteins were further analyzed via hierarchical clustering. Placental lysates of five CTRL and five GDM women were analyzed. (B) Protein-protein interaction network of differentially expressed proteins in placentas of GDM women as compared with CTRL. Nodes represent proteins, and edges represent degrees of connectivity. Enrichment of the node was performed with BINGO, a plug-in for Cytoscape. Rectangular-shaped nodes indicate the upregulated (red) and downregulated (green) proteins in GDM compared with CTRL samples and described to play a role in trophoblasts structure and function (eg, invasion, angiogenesis, oxidative stress, barrier and energy support), fetal development (iron, muscle differentiation and nutrients) and protection from fetal loss (anticoagulant and regulation of epithelial-to-mesenchymal transition). GDM, gestational diabetes mellitus.

and insulin levels, and insulin-like growth factor-1 signaling) (figure 2B). Among the aforementioned proteins detected, PRG2, which controls numerous growth factors activity, was observed as the most highly abundant placental protein in GDM/LGA. Moreover, upregulated placental expression of DPP-4, a key factor in physiological regulation of glucose homeostasis, was also evident in the GDM/LGA cohort. Both PRG2 and DPP-4 protein expressions were confirmed in placental lysates by immunotargeted assays (online supplemental figure $1 \mathrm{~A}$ and $\mathrm{B})$.

\section{Alterations of differentiation markers in hESCs cultured with PRG2 and DPP-4}

To mechanistically understand the role of PRG2 and DPP-4 in fetal development, we cultured human hESC with the recombinant proteins PRG2 and DPP-4 and performed a gene expression profiling of stem cell transcription factors. Transcriptome analysis revealed significant upregulation of HOXD10, a member of the family of Hox genes involved in stem cell differentiation and embryonic limb development, in hESCs exposed to PRG2 (figure 3A). Interestingly, hESCs cultured with DPP-4 exhibited increased expression of cardiac progenitors related genes, TBX5 and IRX4, and decrease of the osteogenic differentiation marker VDR (figure 3B). Since both GDM and GDM/LGA may be associated with an increased risk for newborns to develop metabolic disorders, we explored the effects of PRG2 and DPP-4 on pathways related to hESCs differentiation into mesendoderm (ME) and definitive endoderm (DE), the first steps 
A

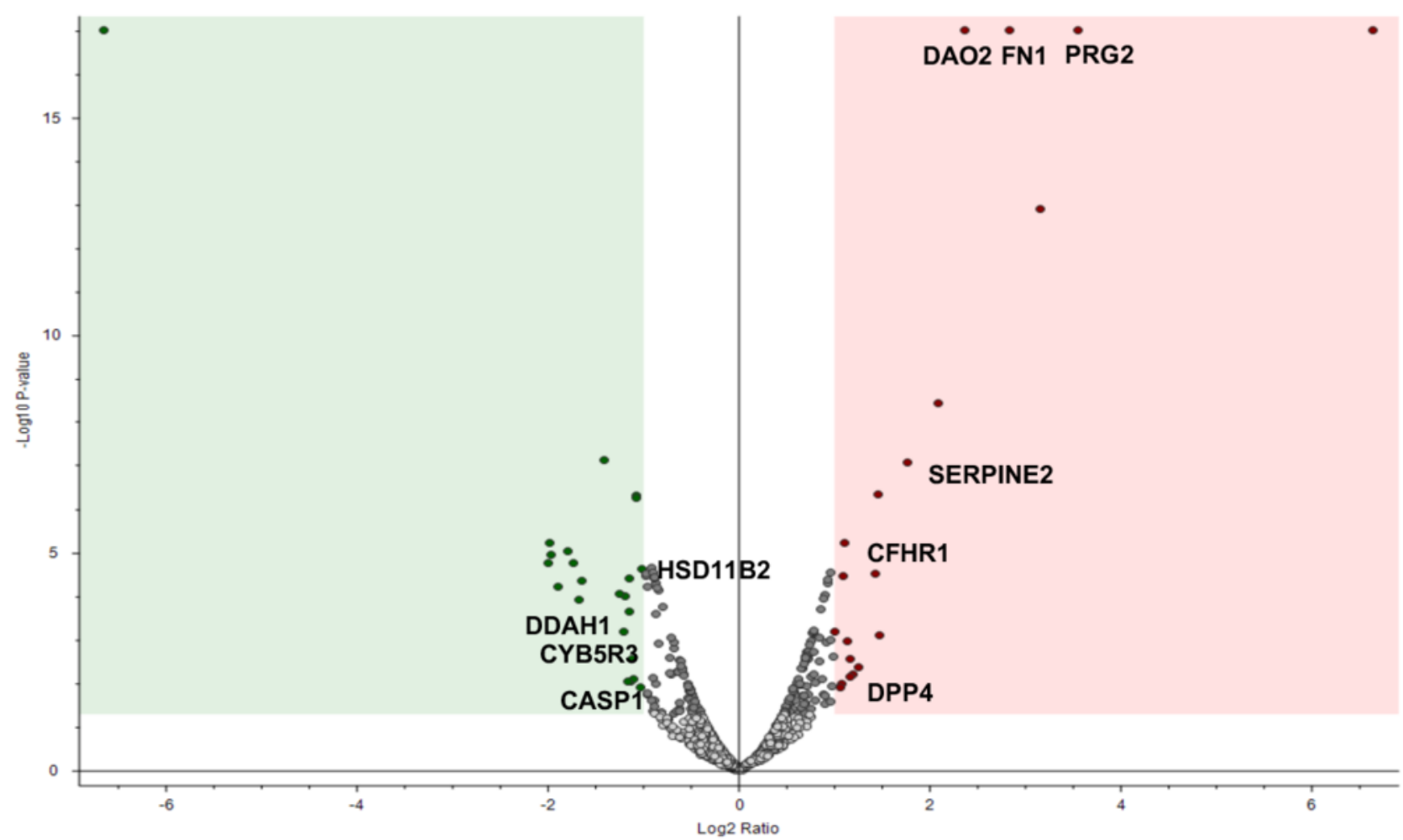

B

\begin{tabular}{ll}
\hline Protein & Function \\
\hline $\begin{array}{l}\text { Upregulated } \\
\text { Bone marrow proteoglycan (PRG2) }\end{array}$ & $\begin{array}{l}\text { Placental regulation of peptide hormone and growth } \\
\text { factor activity }\end{array}$ \\
$\begin{array}{l}\text { Fibronectin (FN1) } \\
\text { Amiloride-sensitive amine oxidase [copper- } \\
\text { containing] (DAO2) }\end{array}$ & $\begin{array}{l}\text { Control of histamine levels during pregnancy } \\
\text { Glia-derived nexin (SERPINE2) }\end{array}$ \\
$\begin{array}{l}\text { Complement factor H-related protein 1 (CFHR1) } \\
\text { Dipeptidyl peptidase 4 (DPP4) }\end{array}$ & Rlacental remodeling \\
\hline $\begin{array}{l}\text { Downregulated } \\
\text { N(G),N(G)-dimethylarginine } \\
\text { dimethylaminohydrolase 1 (DDAH1) }\end{array}$ & Regulation of fetal insulin levels and $\beta$-cell development \\
$\begin{array}{l}\text { NADH-cytochrome b5 reductase 3 (CYB5R3) } \\
\text { Corticosteroid 11-beta-dehydrogenase isozyme 2 } \\
\text { (HSD11B2) }\end{array}$ & Regulation of feto-maternal cortisol levels \\
Caspase-1 (CASP1) & ADMA-NO pathway homeostasis during pregnancy \\
\hline
\end{tabular}

Figure 2 Placental proteomic profile and table of significant upregulated and downregulated proteins in GDM versus GDM/ LGA women. (A) Volcano-plot representing the placental proteomic profile of women with GDM as compared with that of GDM/LGA. Protein's Log2 ratio and -Log10 $p$ values are represented on $x$ and $y$ axes, respectively. Protein in the shaded area represent upregulation (red) and downregulation (green). The complete dataset of identified and quantified proteins was subjected to statistical analysis $(p<0.05)$. Significantly differentially expressed proteins were further analyzed via hierarchical clustering. Placental lysates of four GDM/LGA and four GDM women were analyzed. (B) List of proteins differentially expressed in placentas of GDM as compared with GDM/LGA women and described to play a role in placental inflammation (eg, placental inflammasome, complement regulation, control of nitric oxide and histamine levels during pregnancy), fetal nutrient supply (lipid metabolism) and hormonal homeostasis (cortisol and insulin levels, and insulin-like growth factor-1 signaling). GDM, gestational diabetes mellitus; LGA, large-for-gestational-age. 
A

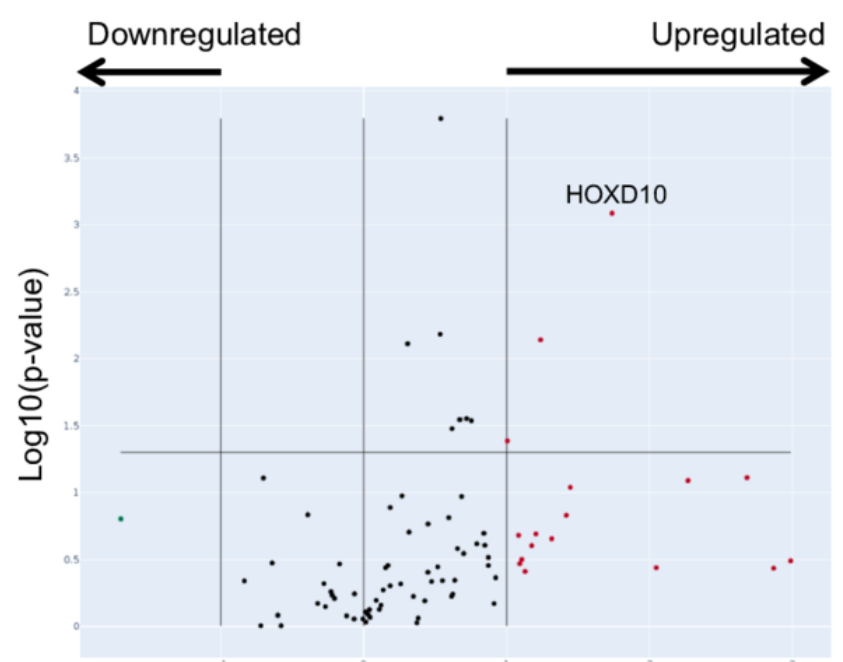

Log2(fold change: PRG2/CTRL)

\begin{tabular}{lll}
\hline Gene Symbol & Fold Regulation & p Value \\
\hline Upregulated & & \\
HOXD10 & 3.34 & 0.000820 \\
\hline
\end{tabular}

B

DPP-4 vs. CTRL

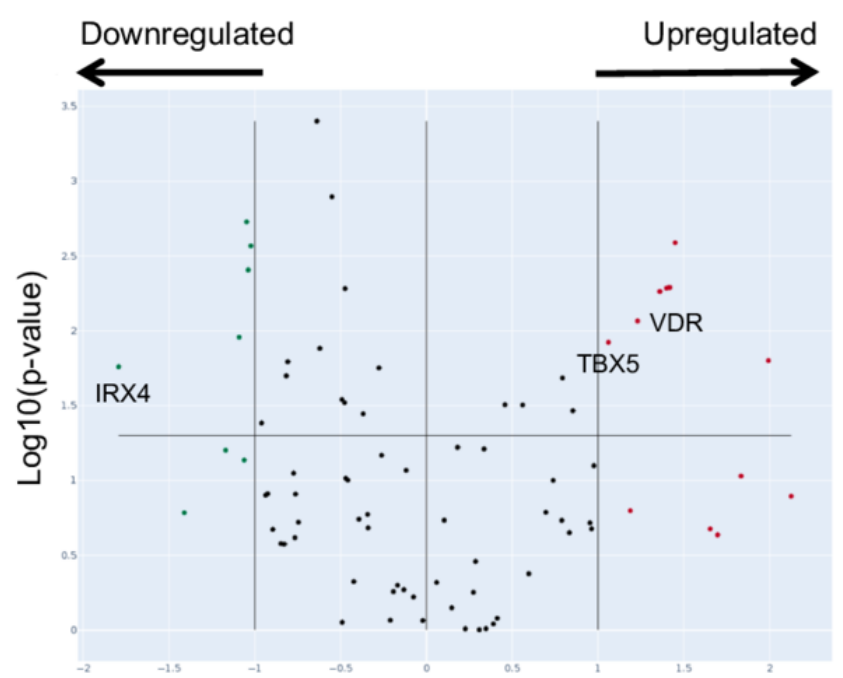

Log2(fold change: DPP-4/CTRL)

\begin{tabular}{lll}
\hline Gene Symbol & Fold Regulation & p Value \\
\hline Upregulated & & \\
TBX5 & 3.98 & 0.015791 \\
VDR & 2.67 & 0.005130 \\
$\quad$ Downregulated & & \\
IRX4 & -3.47 & 0.017416 \\
\hline
\end{tabular}

C

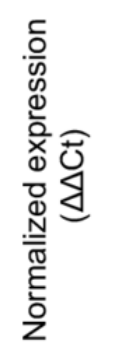

Medium

PRG2 25ug/ml DPP-4 100ng/ml

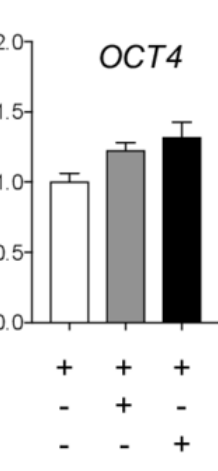

ES

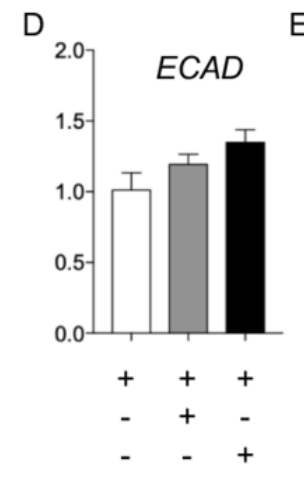

ME

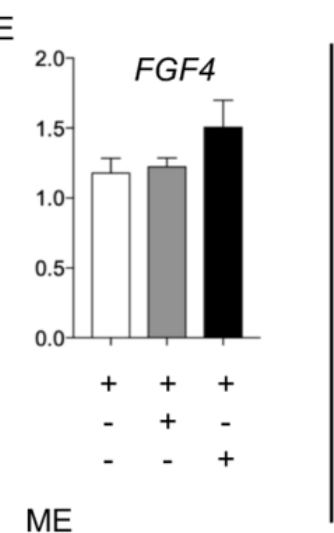

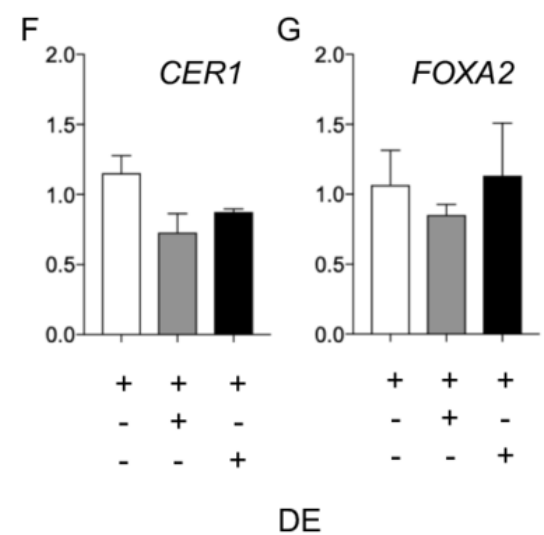

Figure 3 Expression of differentiation markers in hESC cultured with PRG2 or DPP-4. (A and B) volcano plot representing stem cell transcriptome profile of hESC culture with PRG2 (A) and DPP-4 (B) compared with control. Genes' Log2 ratio and Log10 $p$ values are represented on $x$ and y axes, respectively. Upregulated genes are shown in red, and downregulated ones in green. Experiments were performed in triplicate. (C-G) Bar graphs depict expression data of stem cells markers OCT4, ECAD, FGF4, CER, FOXA2 as normalized mRNA expression measured by quantitative RT-PCR on hESC after treatment with PRG2 or DPP-4. All samples were run in triplicate and normalized to expression of the housekeeping gene ACTB ( $\triangle C$ Ct). DE, definitive endoderm; DPP-4, dipeptidyl peptidase-4; ES, hESC; hESC, human embryonic stem cell; ME, mesendoderm; PRG2, bone marrow proteoglycan.

towards pancreatic development. Both PRG2 and DPP-4 increased stem and ME markers OCT4, ECAD and FGF4 in cultured hESCs, while endoderm markers CER1 and FOXA2 was decreased (figure 3C-F), thus suggesting a role in abnormal endocrine pancreas development and in metabolic disorders (ie, type 2 diabetes or obesity) later on. This impairment in endocrine progenitors and early-stage beta cells was further confirmed by the increased expression of NGN3, an endocrine pancreatic progenitor marker, detected in the Beta-lox 5 cell line when cultured in the presence of DPP-4 (online supplemental figure $1 \mathrm{C}$ ).

\section{DISCUSSION}

We are herein describing an altered placental proteome in pregnant women with GDM and more importantly in those with GDM and LGA. Among proteins differentially 
expressed in GDM/LGA, 10 factors were linked to fetal growth/development by acting on hormone homeostasis, energy metabolism and placental nutrient transport, as previous reports suggested. ${ }^{16}$ In particular, PRG2 and DPP-4, found to be upregulated in GDM/LGA placental proteome analysis, are both involved in pregnancy ${ }^{17} 18$ and their dysregulation was shown to correlate with pregnancy-related disorders. ${ }^{19}$ As PRG2 controls several growth factors activity, its upregulation may take part to the abnormal fetal size such as that observed in GDM/LGA, while the DPP-4 increase detected in placenta may account for some growth abnormalities as well as the dysregulated glycometabolic control in the new-borns, which have been shown in literature. ${ }^{1}$ This may be of clinical interest given the availability of DPP-4 inhibitors. ${ }^{20}$ Among proteins found to be altered in GDM/LGA, dimethylarginine dimethylaminohydrolase 1 (DDAH1) was slightly downregulated, which is in line with other reports showing reduced DDAH1 expression and activity in patients with diabetes. ${ }^{21}$ As DDAH1 is responsible for asymmetric dimethylarginine (ADMA) degradation, a decrease in its expression, as that observed in our patients, may suggest an increase in ADMA levels in GDM/LGA patients as observed in other diabetic subjects, thus pointing at placenta as potential site of production in this condition. ${ }^{22}{ }^{23}$ We acknowledge that our study has some limitations, included but not limited to the small number of placenta samples analyzed and the need of a larger validation of the detected proteins.

In order to prove the potential role of PRG2 and DPP-4 in fetal growth, we took advantage of hESCs, a useful tool to assess in vitro effects on fetal development. Our transcriptomic analysis revealed that PRG2 and DPP-4 alter the expression of genes relevant for hESCs differentiation toward cardiac, osteogenic and endodermic progenitors. ${ }^{24}{ }^{25}$ Since DE is the precursor for endocrine pancreas development, alterations on endodermic progenitor markers may suggest an impaired generation of pancreas endocrine compartment and function, thus leading to future metabolic disorders such as obesity and type 2 diabetes, frequently observed as long-term complications in LGA children of GDM mothers. ${ }^{26}$ The mechanisms whereby these proteins may exert their effects on hESCs remains to be established. However, soluble DPP-4 is known to have intact enzymatic activity and it acts in a paracrine or endocrine manner on the targeted cells such as lymphocytes, muscle smooth cells and adipocyte, mainly through the Akt/pAkt, ERK/pERK and/or Nf-kB signaling. ${ }^{27-29}$ Several soluble factors involved in hESC self-renewal and differentiation like LIF, bFGF and BMP4 possess putative truncation site for DPP- 4 and modulation of their levels due to DPP-4 activity could influence hESC physiology. ${ }^{30}$ This has recently been shown as well for SDF-1/CXCR 4 axis that could be modulated by DPP-4 in different types of progenitor cells. ${ }^{31-33}$ Based on these evidences, we hypothesize that DPP-4 may exert its effect on hESC by activating signaling pathways related to hESC differentiation.
In conclusion, our data indicate that the placental proteome is dysregulated in GDM, particularly with LGA fetuses. Among these altered factors, we identified two major factors, PRG2 and DPP-4, related to pregnancy disorders, which control in vitro hESCs differentiation and may in turn directly affect embryonic development. Given the lack of therapeutic opportunities and approaches for GDM and for GDM/LGA, with diet and insulin being the sole treatments approved for these conditions, we thus envision to generate new monoclonal antibodies or fusion-proteins in order to block the activity of the upregulated proteins. DPP-4 inhibitors, available on the market as antidiabetic oral agents, represent an example of a potential inhibitor of DPP-4 enzymatic activity, which may be tested and further validated in large future trial in GDM/LGA.

\section{Author affiliations}

${ }^{1}$ International Center for T1D Pediatric Clinical Research Center Romeo ed Enrica Invernizzi, Department of Biomedical and Clinical Sciences, Università di Milano, Milano, Italy

2"G. Pardi" Laboratory of Maternal-Fetal Translational Research, Department of Biomedical and Clinical Sciences "L. Sacco", Università di Milano, Milano, Lombardia, Italy

${ }^{3}$ Nephrology Division, Boston Children's Hospital, Boston, Massachusetts, USA ${ }^{4}$ Department of Endocrinology, ASST Fatebenefratelli-Sacco, Milano, Lombardia, Italy

${ }^{5}$ ProMiFa, Protein Microsequencing Facility, Ospedale San Raffaele, Milano, Lombardia, Italy

${ }^{6}$ Transplantation Research Center, Renal Division, Brigham \& Women's, Harvard University, Boston, Massachusetts, USA

${ }^{7}$ Faculty of Medicine, Al-Azhar University, Cairo, Egypt

${ }^{8}$ Endocrinologia e Metabolismo, Dipartimento di Scienze della Salute, Universita di Milano, Milano, Italy

${ }^{9}$ Department of Biomedical, Biotechnological and Translational Sciences, Unit of Pathology, University of Parma, Parma, Emilia-Romagna, Italy

${ }^{10}$ Department of Pediatrics, Ospedale dei Bambini V Buzzi, Milano, Lombardia, Italy

Acknowledgements We would like to thank the 'Fondazione Romeo e Enrica Invernizzi' for extraordinary support. Portions of this work were carried out in OMICs, an advanced mass spectrometry platform established by the Università degli Studi di Milano.

Contributors EA, ChM and FD designed the study, performed experiments, analyzed data, and wrote the paper; AM, CL, AJS, MBN, and VU performed experiments and analyzed data; CiM performed proteomic analysis; IP, RA, BEE, DC, FF and GZ coordinated sample collection, designed research and edited the paper; IC and PF designed research, wrote and edited the paper. PF is the guarantor of this work and, as such, had full access to all the data in the study and takes responsibility for the integrity of the data and the accuracy of the data analysis.

Funding FD is the recipient of an SID Lombardia Grant. PF is the recipient of the Italian Ministry of Health RF-2016-02362512 grant. VU is supported by a F0.DI.RI SID fellowship.

Competing interests None declared.

Patient consent for publication Not required.

Ethics approval The study protocol number 52/2010 was approved by the ASST Fatebenefratelli-Sacco Ethic Committee. Each individual enrolled provided informed consent to participate in the study.

Provenance and peer review Not commissioned; externally peer reviewed.

Data availability statement Data are available on reasonable request. All data relevant to the study are included in the article or uploaded as supplemental information. The datasets used and/or analyzed during the current study are available from the corresponding author on reasonable request. 
Supplemental material This content has been supplied by the author(s). It has not been vetted by BMJ Publishing Group Limited (BMJ) and may not have been peer-reviewed. Any opinions or recommendations discussed are solely those of the author(s) and are not endorsed by BMJ. BMJ disclaims all liability and responsibility arising from any reliance placed on the content. Where the content includes any translated material, BMJ does not warrant the accuracy and reliability of the translations (including but not limited to local regulations, clinical guidelines, terminology, drug names and drug dosages), and is not responsible for any error and/or omissions arising from translation and adaptation or otherwise.

Open access This is an open access article distributed in accordance with the Creative Commons Attribution Non Commercial (CC BY-NC 4.0) license, which permits others to distribute, remix, adapt, build upon this work non-commercially, and license their derivative works on different terms, provided the original work is properly cited, appropriate credit is given, any changes made indicated, and the use is non-commercial. See: http://creativecommons.org/licenses/by-nc/4.0/.

ORCID iDs

Franco Folli http://orcid.org/0000-0001-9824-5222

Paolo Fiorina http://orcid.org/0000-0002-1093-7724

\section{REFERENCES}

1 Denney JM, Quinn KH. Gestational diabetes: underpinning principles, surveillance, and management. Obstet Gynecol Clin North Am 2018;45:299-314.

2 Metzger BE, Lowe LP, HAPO Study Cooperative Research Group, et al. Hyperglycemia and adverse pregnancy outcomes. $N$ Engl J Med 2008;358:1991-2002.

3 Jensen DM, Damm P, Moelsted-Pedersen L, et al. Outcomes in type 1 diabetic pregnancies: a nationwide, population-based study. Diabetes Care 2004;27:2819-23.

4 Riella LV, Dada S, Chabtini L, et al. B7h (ICOS-L) maintains tolerance at the fetomaternal interface. Am J Pathol 2013;182:2204-13.

5 Mandò C, Anelli GM, Novielli C, et al. Impact of obesity and hyperglycemia on placental mitochondria. Oxid Med Cell Longev 2018;2018:1-10.

6 D'Addio F, La Rosa S, Maestroni A, et al. Circulating IGF-I and IGFBP3 levels control human colonic stem cell function and are disrupted in diabetic enteropathy. Cell Stem Cell 2015;17:486-98.

7 Folli F, Guzzi V, Perego L, et al. Proteomics reveals novel oxidative and glycolytic mechanisms in type 1 diabetic patients' skin which are normalized by kidney-pancreas transplantation. PLoS One 2010;5:e9923.

8 Liu B, Xu Y, Voss C, et al. Altered protein expression in gestational diabetes mellitus placentas provides insight into insulin resistance and coagulation/fibrinolysis pathways. PLoS One 2012;7:e44701.

9 Wang F, Shi Z, Wang P, et al. Comparative proteome profile of human placenta from normal and preeclamptic pregnancies. PLoS One 2013;8:e78025.

10 Lapolla A, Porcu S, Roverso M, et al. A preliminary investigation on placenta protein profile reveals only modest changes in well controlled gestational diabetes mellitus. Eur J Mass Spectrom 2013;19:211-23.

11 Roverso M, Brioschi M, Banfi C, et al. A preliminary study on human placental tissue impaired by gestational diabetes: a comparison of gel-based versus gel-free proteomics approaches. Eur J Mass Spectrom 2016;22:71-82.

12 Hod M, Kapur A, Sacks DA, et al. The International Federation of Gynecology and Obstetrics (FIGO) Initiative on gestational diabetes mellitus: A pragmatic guide for diagnosis, management, and care. International Journal of Gynecology \& Obstetrics 2015;131:S173-211.
13 Wiśniewski JR, Zougman A, Nagaraj N, et al. Universal sample preparation method for proteome analysis. Nat Methods 2009;6:359-62.

14 Maere S, Heymans K, Kuiper M. BiNGO: a Cytoscape plugin to assess overrepresentation of gene ontology categories in biologica networks. Bioinformatics 2005;21:3448-9.

15 D'Addio F, Vergani A, Potena L, et al. P2X7R mutation disrupts the NLRP3-mediated th program and predicts poor cardiac allograft outcomes. J Clin Invest 2018;128:3490-503.

16 Cetin I, de Santis MSN, Taricco E, et al. Maternal and fetal amino acid concentrations in normal pregnancies and in pregnancies with gestational diabetes mellitus. Am J Obstet Gynecol 2005;192:610-7.

17 Weyer K, Glerup S. Placental regulation of peptide hormone and growth factor activity by proMBP. Biol Reprod 2011;84:1077-86.

18 Imai K, Kanzaki H, Fujiwara $\mathrm{H}$, et al. Expression and localization of aminopeptidase $\mathrm{N}$, neutral endopeptidase, and dipeptidyl peptidase IV in the human placenta and fetal membranes. Am J Obstet Gynecol 1994;170:1163-8.

19 Kandzija N, Zhang W, Motta-Mejia C, et al. Placental extracellular vesicles express active dipeptidyl peptidase IV; levels are increased in gestational diabetes mellitus. J Extracell Vesicles 2019;8:1617000.

20 Malvandi AM, Loretelli C, Ben Nasr M, et al. Sitagliptin favorably modulates immune-relevant pathways in human beta cells. Pharmacol Res 2019;148:104405.

21 Wetzel MD, Gao T, Stanley K, et al. Enhancing kidney DDAH1 expression by adenovirus delivery reduces ADMA and ameliorates diabetic nephropathy. Am J Physiol Renal Physiol 2020;318:F509-17.

22 Lajer M, Tarnow L, Jorsal A, et al. Plasma concentration of asymmetric dimethylarginine (ADMA) predicts cardiovascular morbidity and mortality in type 1 diabetic patients with diabetic nephropathy. Diabetes Care 2008;31:747-52.

23 Cighetti G, Fermo I, Aman CS, et al. Dimethylarginines in complicated type 1 diabetes: roles of insulin, glucose, and oxidative stress. Free Radic Biol Med 2009;47:307-11.

24 Nelson DO, Lalit PA, Biermann M, et al. Irx4 marks a multipotent, Ventricular-Specific progenitor cell. Stem Cells 2016;34:2875-88.

25 Olivares-Navarrete R, Sutha K, Hyzy SL, et al. Osteogenic differentiation of stem cells alters vitamin $\mathrm{D}$ receptor expression. Stem Cells Dev 2012;21:1726-35.

26 Johnsson IW, Haglund B, Ahlsson F, et al. A high birth weight is associated with increased risk of type 2 diabetes and obesity. Pediatr Obes 2015;10:77-83.

27 Röhrborn D, Wronkowitz N, Eckel J. Dpp4 in diabetes. Front Immunol 2015:6:386.

28 Lamers D, Famulla S, Wronkowitz N, et al. Dipeptidyl peptidase 4 is a novel adipokine potentially linking obesity to the metabolic syndrome. Diabetes 2011;60:1917-25.

29 Yu DMT, Slaitini L, Gysbers V, et al. Soluble CD26 / dipeptidyl peptidase IV enhances human lymphocyte proliferation in vitro independent of dipeptidyl peptidase enzyme activity and adenosine deaminase binding. Scand J Immunol 2011;73:102-11.

$30 \mathrm{Ou}$ X, O'Leary HA, Broxmeyer HE. Implications of DPP4 modification of proteins that regulate stem/progenitor and more mature cell types. Blood 2013;122:161-9.

31 Fadini GP, Boscaro E, Albiero M, et al. The oral dipeptidyl peptidase-4 inhibitor sitagliptin increases circulating endothelial progenitor cells in patients with type 2 diabetes: possible role of stromal-derived factor-1alpha. Diabetes Care 2010;33:1607-9.

32 Zhong J, Rajagopalan S. Dipeptidyl peptidase-4 regulation of SDF-1/ CXCR4 axis: implications for cardiovascular disease. Front Immunol 2015;6:477

33 Deacon CF. Corrigendum: physiology and pharmacology of DPP-4 in glucose homeostasis and the treatment of type 2 diabetes. Front Endocrinol 2019;10:275 